\title{
Intrapulse x-ray parametric amplification in high-order-harmonic generation
}

\author{
Carles Serrat* \\ Universitat Politècnica de Catalunya, Colom 11, 08222 Terrassa, Barcelona, Spain
}

(Received 20 April 2016; published xxxxxx)

\begin{abstract}
We demonstrate strong-field-driven impulsive XUV-x-ray parametric amplification (IXPA) processes in high-order harmonic generation at the single-atom level by using ab initio calculations. We consider the example of $\mathrm{Li}^{+}$ions exposed simultaneously to an intense IR pulse and a weak 200-as XUV-X-ray pulse with central photon energies varying from 90 to $400 \mathrm{eV}$. We determine optimal parameter ranges and the precise delays between the IR and the XUV-x-ray pulses for IXPA to occur. The present results might be a guide to achieve exponential growth of the XUV-X-ray signal in tabletop XUV-X-ray lasers.
\end{abstract} 4 light pulses can be generated in tabletop high-order har15 monic generation (HHG) sources. Important applications 16 suffer, however, from the low efficiency inherent to HHG 17 processes. Different techniques have been investigated to over18 come this low efficiency and important advances have been made [1-8]. Strong-field assisted XUV-X-ray parametric amplification processes in particular are investigated since they can produce avalanche effects in the medium that result in an exponential growth of the XUV-X-ray signal [9-15].

In previous research we addressed the influence of weak XUV radiation pulses of photon energies far from the ionization potential of the medium added to the strong IR driving pulse in $\mathrm{HHG}$ processes $[11,13,14,16,17]$. In particular, we showed that forward scattering can be largely enhanced when an XUV pulse is optimally synchronized with the IR pulse [11]. This theoretical prediction was soon corroborated by experiments [12] and a further detailed comparison between the theory and the experimental measurements showed good qualitative agreement [14]. Strong-field-mediated intrapulse Xray parametric amplification (IXPA) processes were identified in [14] as decisive for the amplification at the single-atom level. The theory used in these previous studies was based on the strong-field approximation (SFA) [18]. It is therefore important to investigate the effect of the Coulomb potential of the atom, which is basically neglected in the SFA, on the amplification effects.

In this paper we perform time-dependent Schrödinger 41 equation (TDSE) simulations in one dimension [19-21], which 42 correctly consider the effect of the Coulomb potential, and 3 demonstrate the IXPA effect using an $a b$ initio theory by 44 considering the $\mathrm{Li}^{+}$ion $\left(I_{p}=75.6 \mathrm{eV}\right)$ as the amplifying 45 medium. We have chosen to investigate optimal parameter 46 ranges for IXPA in $\mathrm{Li}^{+}$for two reasons. First, parametric $47 \mathrm{XUV}-\mathrm{X}$-ray amplification effects were shown to benefit at 48 the single-atom level by high gas ionization potentials [13]. 49 Harmonics with large contrast were predicted compared to the 50 broad spectrum obtained from the lower ionization potentials 1 of gases as He. Second, a high ionization potential will 52 also favor the avalanche effect produced by the parametric 53 amplification processes [14], because those need a high

\footnotetext{
*carles.serrat-jurado@upc.edu
}

degree of synchronization between the XUV-X-ray and IR 54 pulses, and this synchronization is optimally preserved by the 55 lower plasma densities produced in higher ionization potential 56 media. The results that we present might hence be a first 57 valuable guide for future experiments. In our simulations, we 58 use the soft-Coulomb potential

$$
V(x)=-\frac{1}{\sqrt{x^{2}+\beta^{2}}},
$$

where we use atomic units and $\beta^{2}=0.0252$. The IR field is 60 a 800 -nm wavelength, 4 -fs $\sin ^{2}$ pulse with a peak intensity 61 of $2.5 \times 10^{15} \mathrm{~W} / \mathrm{cm}^{2}$, which produces a maximum of $\approx 0.2 \% \quad 62$ ground-state depletion. The seed XUV-X-ray pulse is a $\sin ^{2}{ }_{63}$ pulse with a peak intensity of $2.5 \times 10^{11} \mathrm{~W} / \mathrm{cm}^{2}$ and a duration 64 of 200 as. IXPA processes are sensitive to the different 65 parameters involved in the interaction process, such as the 66 intensity of the IR pulse and the associated ground-state 67 depletion, as well as the duration and the frequency content 68 of the weak XUV-X-ray attosecond pulse. In what follows we 69 study the influence of the XUV-X-ray central photon energy 70 together with the delay with respect to the IR pulse on the 71 IXPA effect at the single-atom level.

Figure 1 shows a basic scheme for IXPA processes. 73 The low-frequency region of the XUV-X-ray weak pulse is 74 absorbed by the electron, a first step that is assisted by the 75 presence of the strong IR driving pulse that modifies the 76 Coulomb potential of the ion; in a second step the electron 77 is accelerated in the continuum by the IR electric field, 78 reaching the necessary energy at the correct time so that a 79 higher-frequency region of the XUV-X-ray pulse, optimally 80 the central frequency region, stimulates the emission in a third 81 step. The process is produced in the time scale of the duration 82 of the XUV-X-ray pulse and benefits from the reshaping (chirp) 83 during the interaction with the medium, as will be further 84 detailed below.

In order to describe IXPA processes it is useful to compute 86 the XUV-X-ray probe absorption spectrum, as described 87 in [22], which represents the absorption of a weak probe pulse 88 following a nonlinear excitation by one or more pump laser 89 pulses

90

$$
S(\omega) \propto \operatorname{Im}\left[E_{X}^{*}(\omega) D_{X}(\omega)\right] .
$$

To probe the state of our system due to the nonlinear excitation 91 caused by the presence of both the strong IR field and the 92 




intrapulse X-Ray parametric amplification

FIG. 1. Schematic of a strong-field-mediated IXPA.

93

94 dipole acceleration obtained in the TDSE integration the

95 response of the system to the IR field alone and hence purely

$96 \mathrm{HHG}$ processes are not included in the time-dependent dipole 97 acceleration that plays the role of the pump in Eq. (2). We 98 therefore define $D_{X}(\omega)=D(\omega)-D_{\mathrm{IR}}(\omega)$, where $D(\omega)$ is the 99 complete spectrum obtained from the excitation of the system 100 and $D_{\mathrm{IR}}(\omega)$ is the spectrum corresponding to the excitation 01 of the system with the IR field alone. In this way, $S(\omega)$ 102 calculates the absorption that a weak probe pulse $E_{X}^{*}(\omega)$ would 103 experience interacting with a system that has been pumped 104 and presents the time-dependent dipole acceleration that gives 105 the spectrum $D_{X}(\omega)$, which includes the linear response to 106 the weak XUV-X-ray pulse. The probe pulse $E_{X}^{*}(\omega)$ in our 107 calculations is the seed that we consider for the combined IR 108 plus XUV-X-ray interaction.

109 Figure 2 shows the probe absorption spectra for the case of 110 a 113-eV XUV pulse interacting with the ion together with the

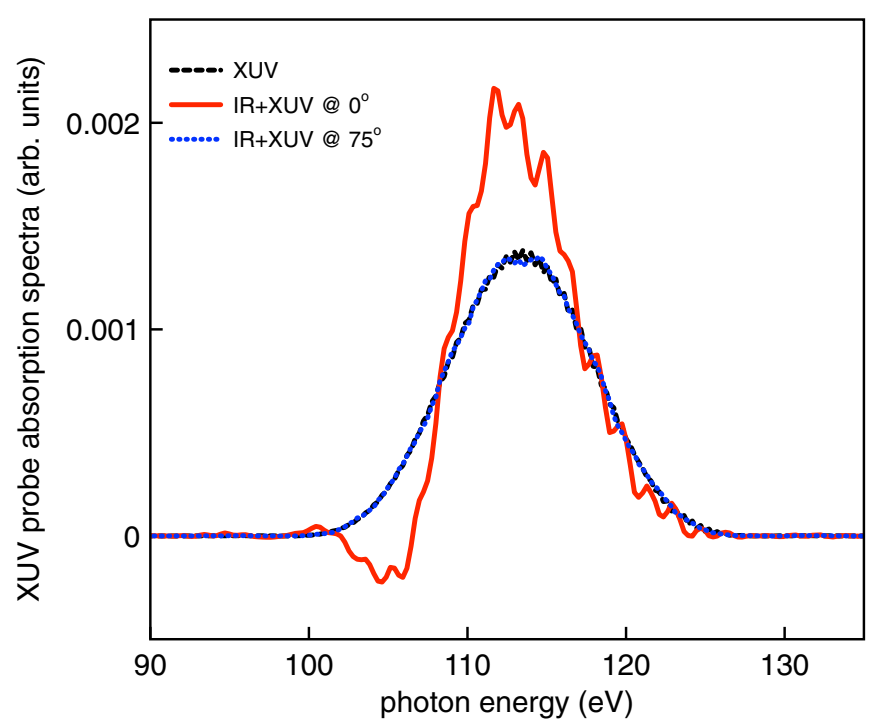

FIG. 2. XUV-X-ray probe absorption spectrum for a $113-\mathrm{eV}$ XUV pulse interacting together with the IR pulse at a delay of $0^{\circ}$ (red solid line), and $75^{\circ}$ (blue dotted line), as indicated. The black dashed line shows the probe absorption spectrum in the case that the XUV pulse interacts alone with the ion.
IR pulse at two different delays. The red curve shows the case 111 that the XUV pulse and the IR pulse are synchronized at a delay 112 of $0^{\circ}$ and the blue dotted curve shows the case for which the ${ }_{113}$ delay is equal to $75^{\circ}$. The results are compared to the case for 114 which the weak XUV pulse interacts alone with the ion (black 115 dashed curve), which gives a purely linear response. From the 116 calculated probe absorption spectra (Fig. 2), it is clear that only 117 certain delays between the IR and the XUV pulses can produce ${ }_{118}$ an effect different from the linear response. The red curve at ${ }_{119}$ a delay of $0^{\circ}$ shows probe emission (negative values) at the ${ }_{120}$ lower frequencies of the XUV pulse, around $105 \mathrm{eV}$, which 121 means that the probe pulse finds the system in an excited state, 122 producing stimulated emission and hence probe gain at this 123 frequency range. The red curve in Fig. 2 also shows probe ${ }_{124}$ absorption (positive values) around the central frequency of 125 the XUV pulse that overcomes the probe absorption calculated 126 for the purely linear case (black line), which means that the ${ }_{127}$ stimulated emission produced in this region by the IXPA 128 process has depleted the excited state as compared to the linear 129 case and therefore the probe absorption is higher. Indeed, the ${ }_{130}$ probe absorption spectral shape shown by the red line in Fig. $2 \quad 131$ is a signature of an intrapulse parametric process.

For a full characterization of the parametric processes we ${ }_{133}$ use two more tools. (i) We compare the spectra from the 134 response of the system to the IR pulse alone, the XUV- ${ }_{135}$ $\mathrm{X}$-ray pulse alone, and the combined IR plus XUV-X-ray ${ }_{136}$ pulses considering different delays between them. (ii) We ${ }_{137}$ perform a frequency-time analysis to determine at which times 138 the signals are emitted. Furthermore, in the frequency-time 139 analysis, we identify the IR-driven stimulated emission and 140 absorption processes by removing the contributions from 141 the signal produced by the IR pulse alone and the one 142 produced by the XUV-X-ray pulse alone. As it will be further ${ }_{143}$ discussed below, this analysis allows us to determine the 144 parameter regions where IR-driven parametric amplification 145 in the spectral range of the seeded XUV-X-ray pulse occurs. $\quad{ }_{146}$

First, in order to determine the parameter ranges where ${ }_{147}$ nonlinear stimulated absorption occurs, we have calculated 148 the integral of the negative regions of the XUV-X-ray probe 149 absorption spectrum by varying both the XUV-X-ray central 150 photon energy from $90 \mathrm{eV}$ to $400 \mathrm{eV}$ and the delay between the 151 XUV-X-ray pulse and the IR field between $-180^{\circ}$ and $180^{\circ} .152$ The resulting map (Fig. 3) illustrates where the IXPA processes 153 most probably arise, since nonlinear stimulated absorption is 154 a necessary step to produce them. Note that Fig. 3 nicely 155 reproduces the shape of the IR electric field as it was already 156 observed in [14]. The particular cases shown in Fig. 2 hence 157 correspond to the 113-eV photon energy value in Fig. 3 at the 158 delays $0^{\circ}$ and $75^{\circ}$.

Figure 4 shows the output spectra obtained for the particular 160 case shown in Fig. 2 around $113 \mathrm{eV}$. The black dashed line ${ }_{161}$ in Fig. 4 corresponds to the spectrum obtained with only 162 the strong IR pulse and the blue dotted line is the spectrum 163 obtained with only the $113-\mathrm{eV}$ XUV pulse. We note that the 164 HHG yield produced by the $\mathrm{Li}^{+}$ion is about four orders 165 of magnitude less than the linear response of the ion to the 166 XUV pulse alone. The red line in Fig. 4 shows the spectrum 167 obtained when both the XUV and IR pulses are sent together 168 and synchronized at a delay of $0^{\circ}$. We can see that the spectral 169 shape nicely follows the characteristics of an IXPA process, 170 




FIG. 3. Integrated negative regions of the XUV-X-ray probe absorption spectra as a function of the central photon energy of the XUV-X-ray pulse and the delay between the IR and XUV-X-ray pulse.

171 as it was also determined by the probe absorption spectrum in 172 Fig. 2. We observe the absorption of the energy around 105 $173 \mathrm{eV}$ and also the amplification of the signal beyond the linear 174 response about the central frequency of the XUV pulse. When 175 the XUV pulse is not optimally synchronized with the IR 176 pulse, however, the nonlinear effects disappear and we obtain 177 basically the same probe absorption spectrum (blue line in 178 Fig. 2) and output spectrum (green line in Fig. 4) that are 179 obtained by considering the interaction with the XUV pulse 180 alone.

181 Figure 5 shows the frequency-time analysis of some of 182 the spectra calculated by considering the results in Fig. 3. 183 Figures 5(a) and 5(b) show the spectra at $113 \mathrm{eV}$ for a 184 delay of $0^{\circ}$ and Figs. 5(c) and 5(d) correspond to the higher

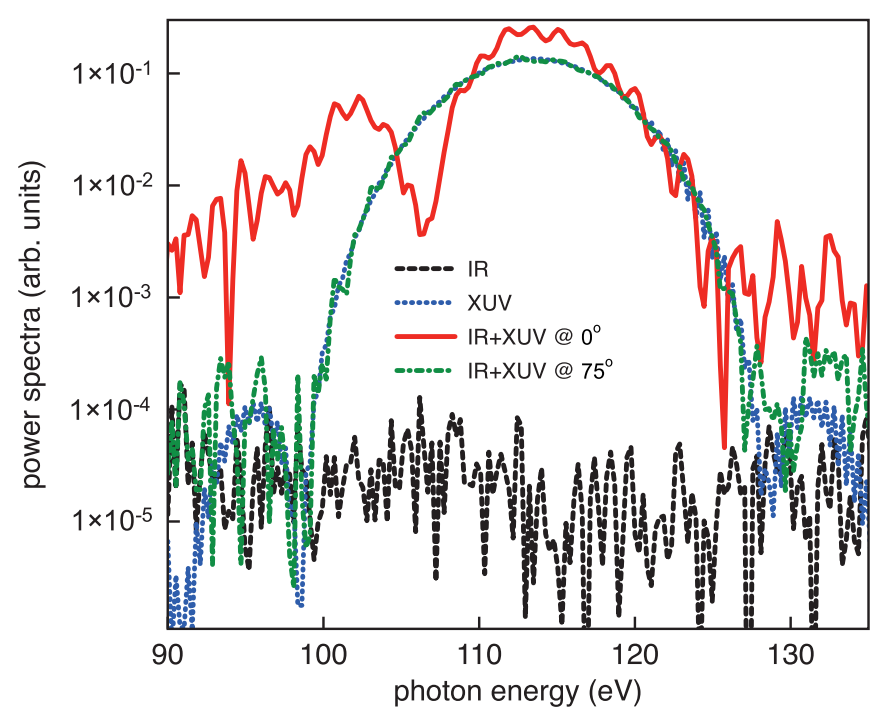

FIG. 4. Output spectra obtained from the Fourier transform of the time-dependent dipole acceleration, around the 113-eV region and for four different interaction cases, as indicated.
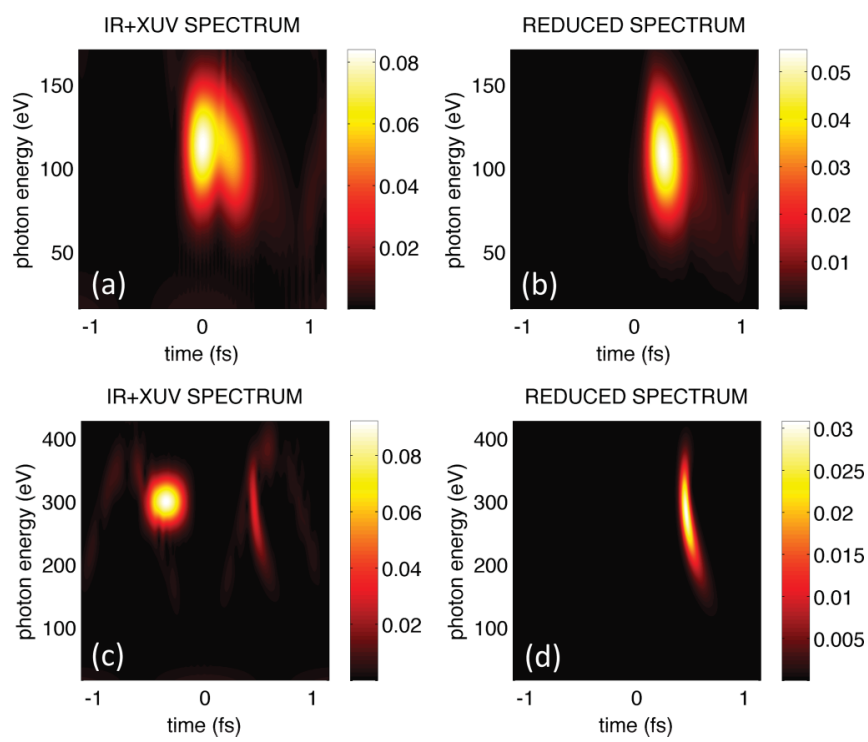

FIG. 5. Frequency-time analysis. (a) and (b) Spectra around $113 \mathrm{eV}$ for a delay of $0^{\circ}$. (c) and (d) Spectra around $300 \mathrm{eV}$ for a delay of $-50^{\circ}$. The reduced spectra in (b) and (d) are obtained by subtracting the response of the IR pulse alone and the one of the XUV pulse alone from the total spectra, as explained in the text.

photon energy of $300 \mathrm{eV}$ and for a delay of $-50^{\circ}$. Both cases 185 correspond to areas in Fig. 3 where IXPA is expected to occur. 186 Figure 5(a) shows the frequency-time spectra for the full IR ${ }_{187}$ plus XUV response around the 113-eV spectral region. In this 188 figure we can observe both the effect of the linear response at 189 the position where the XUV has been added, i.e., at a delay of 190 $0^{\circ}(t=0)$, and the nonlinear response around $t \approx 0.2$ fs. As 191 already commented above, it is worth noting that the 200-as 192 XUV pulse is chirped due to dispersion during the interaction 193 with the ion and consequently time broadened, which allows 194 the IXPA processes to be produced at $t \approx 0.2 \mathrm{fs}$ in this case. In 195 Fig. 5(b) we plot the spectrum resulting from removing from 196 the total response the one due to the IR pulse alone and the one ${ }_{197}$ due to the XUV pulse alone so that we remove the pure HHG ${ }_{198}$ contribution and the contribution of the linear response of the 199 medium to the XUV pulse. We call it the reduced spectrum 200 in Figs. 5(b) and 5(d) and hence it only shows the nonlinear 201 contribution due to the combination of IR and XUV-X-ray 202 assisted effects. We note that the amplified signal [Fig. 5(b)] is 203 slightly down chirped. A similar behavior is shown in Figs. 5(c) 204 and 5(d) by considering a higher-photon-energy X-ray seed 205 pulse of 300-eV central photon energy. In this case the optimal 206 delay, as calculated in Fig. 3 , is about $-50^{\circ}(t \approx-0.4 \mathrm{fs})$. The 207 nonlinear amplified IXPA signal is produced in this case at 208 $t \approx 0.5 \mathrm{fs}$, which again shows that the initial transform-limited 209 $\mathrm{X}$-ray seed is time broadened during the interaction with the 210 $\mathrm{Li}^{+}$ion.

Finally, we address the effect of the added XUV-X-ray 212 pulse (by taking as an example the $113 \mathrm{eV}$ seed pulse) on 213 the larger photon energy regions of the HHG spectrum. As 214 shown in Fig. 6, a considerable increase of the HHG yield is 215 obtained in the case of adding a seed pulse at $113 \mathrm{eV}$, with a 216 plateau of harmonics that ends in this case at about $400 \mathrm{eV} .217$ We observe that this enhancement of the HHG yield depends 218 


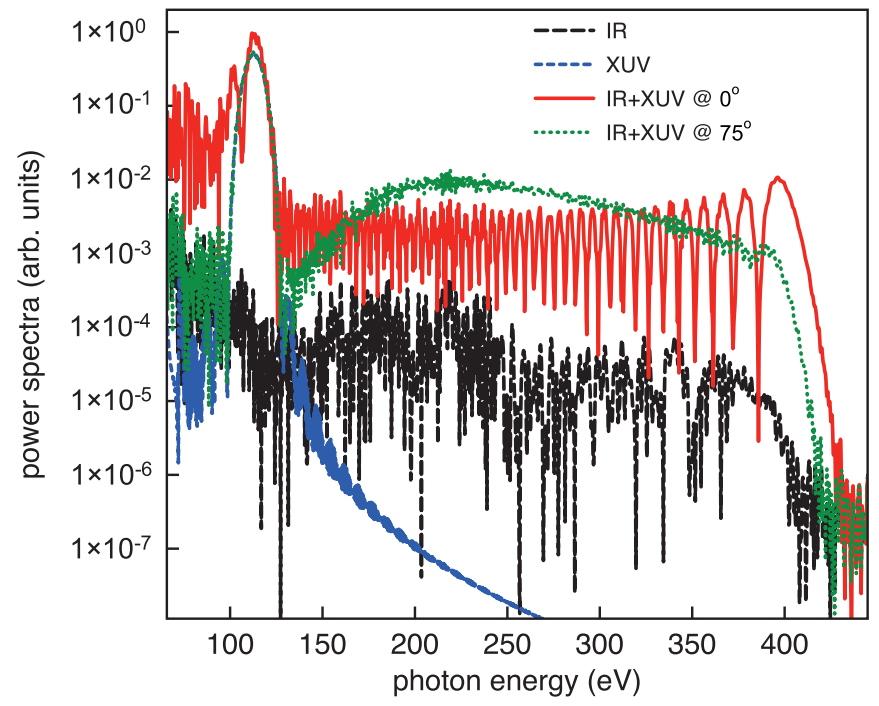

FIG. 6. Spectra obtained from the Fourier transform of the timedependent dipole acceleration for the spectral region between 90 and $450 \mathrm{eV}$ for four different interaction cases, as indicated.

219 on the delay between the XUV and the IR pulse, as it is clear 220 from Fig. 6. Indeed, the red line in Fig. 6 shows spectrally 221 separated harmonics and corresponds to a delay of $0^{\circ}$, while the green line corresponds to a delay of $75^{\circ}$ and shows a ${ }_{222}$ basically flat plateau. This behavior clearly corresponds to 223 enhanced ionization phenomena that have been extensively 224 studied [23-25]. Importantly, although it is not the central 225 subject of this paper, we observe that the enhanced ionization 226 effect is highly robust for the parameter ranges that have been ${ }_{227}$ here considered.

We have hence demonstrated IXPA processes in high-order 229 harmonic generation at the single-atom level by using ab initio 230 simulations and have determined optimal parameter ranges for ${ }_{231}$ IXPA processes to occur. Our study corroborates previous 232 theoretical work performed under the SFA $[11,13,14]$. We 233 conclude that several parameters are decisive and need to be ${ }_{234}$ balanced for IXPA processes to arise, such as the peak intensity 235 of the driving IR pulse, the ionization potential of the medium, 236 and the duration and spectral contents of the weak XUV-X-ray ${ }_{237}$ seed pulses. As already clear from previous studies, the delay ${ }_{238}$ between the weak seed and strong driving pulses is crucial 239 for the amplification. The avalanche effects expected from 240 IXPA processes when propagation and macroscopic effects are 241 considered [14] result in exponential growth of the XUV-X-ray 242 signal, which might be of importance for the implementation 243 of intense tabletop XUV-X-ray lasers and also used as spectral 244 narrow seeds for fully coherent plasma X-ray amplifiers. $\quad 245$

Financial support from the Spanish Ministry of Economy 246 and Competitiveness (FIS2014-51997-R) is acknowledged. $\quad{ }_{247}^{24}$
[1] P. Agostini and L. F. DiMauro, Rep. Prog. Phys. 67, 813 (2004).

[2] T. Popmintchev, M.-C. Chen, D. Popmintchev, P. Arpin, S. Brown, S. Alisauskas, G. Andriukaitis, T. Balciunas, O. D. Mücke, A. Pugzlys, A. Baltuska, B. Shim, S. E. Schrauth, A. Gaeta, C. Hernández-García, L. Plaja, A. Becker, A. JaronBecker, M. M. Murnane, and H. C. Kapteyn, Science 336, 1287 (2012).

[3] C. Serrat and J. Biegert, Phys. Rev. Lett. 104, 073901 (2010).

[4] E. J. Takahashi, P. Lan, O. D. Mücke, Y. Nabekawa, and K. Midorikawa, Nat. Commun. 4, 2691 (2013).

[5] F. Brizuela, C. M. Heyl, P. Rudawski, D. Kroon, L. Rading, J. M. Dahlstrom, J. Mauritsson, P. Johnsson, C. L. Arnold, and A. L'Huillier, Sci. Rep. 3, 1410 (2013).

[6] S. Haessler, T. Balciunas, G. Fan, G. Andriukaitis, A. Pugzlys, A. Baltuska, T. Witting, R. Squibb, A. Zair, J. W. G. Tisch, J. P. Marangos, and L. E. Chipperfield, Phys. Rev. X 4, 021028 (2014).

[7] C. Jin, G. Wang, H. Wei, A.-T. Le, and C. D. Lin, Nat. Commun. 5, 4003 (2014)

[8] L. V. Dao, Kh. B. Dinh, and P. Hannaford, Nat. Commun. 6, 7175 (2015).

[9] J. Seres, E. Seres, D. Hochhaus, B. Ecker, D. Zimmer, V. Bagnoud, T. Kuehl, and C. Spielmann, Nat. Phys. 6, 455 (2010).

[10] J. Seres, E. Seres, and C. Spielmann, Phys. Rev. A 86, 013822 (2012).

[11] C. Serrat, Phys. Rev. Lett. 111, 133902 (2013).
[12] J. Seres, E. Seres, B. Landgraf, B. Ecker, B. Aurand, T. Kuehl, and C. Spielmann, Sci. Rep. 4, 4234 (2014).

[13] C. Serrat, D. Roca, and J. Seres, Opt. Express 23, 4867 (2015).

[14] C. Serrat, D. Roca, J. M. Budesca, J. Seres, E. Seres, B. Aurand, A. Hoffmann, S. Namba, T. Kuehl, and C. Spielmann, Opt. Express 24, 8028 (2016)

[15] T. Bredtmann, S. Chelkowski, A. D. Bandrauk, and M. Ivanov, Phys. Rev. A 93, 021402(R) (2016).

[16] C. Serrat, Appl. Sci. 2, 816 (2012).

[17] C. Serrat, Phys. Rev. A 87, 013825 (2013).

[18] M. Lewenstein, P. Balcou, M. Y. Ivanov, A. L'Huillier, and P. B. Corkum, Phys. Rev. A 49, 2117 (1994).

[19] G. N. Gibson and J. Biegert, Phys. Rev. A 78, 033423 (2008).

[20] E. Hijano, C. Serrat, G. N. Gibson, and J. Biegert, Phys. Rev. A 81, 041401(R) (2010)

[21] E. Hijano, C. Serrat, G. N. Gibson, C. Figueira de Morisso Faria, and J. Biegert, J. Mod. Opt. 58, 1166 (2011).

[22] S. Mukamel, in Principles of Nonlinear Optical Spectroscopy (Oxford University Press, Oxford, 1995), p. 103.

[23] A. D. Bandrauk and N. H. Shon, Phys. Rev. A 66, 031401(R) (2002).

[24] J. Biegert, A. Heinrich, C. P. Hauri, W. Kornelis, P. Schlup, M. P. Anscombe, M. B. Gaarde, K. J. Schafer, and U. Keller, J. Mod. Opt. 53, 87 (2006).

[25] M. Tudorovskaya and M. Lein, J. Mod. Opt. 61, 845 (2014). 